Article

\title{
Identification and Prevalence of Phascolarctid \\ Gammaherpesvirus Types 1 and 2 in South Australian Koala Populations
}

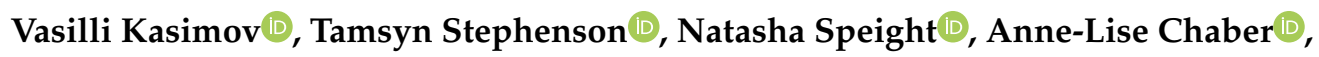 \\ Wayne Boardman ${ }^{(D)}$, Ruby Easther and Farhid Hemmatzadeh *(D) \\ School of Animal and Veterinary Sciences, The University of Adelaide, Roseworthy campus, \\ 5371 Adelaide, Australia; vasilli.kasimov@hotmail.com (V.K.); tamsyn.stephenson@adelaide.edu.au (T.S.); \\ natasha.speight@adelaide.edu.au (N.S.); anne-lise.chaber@adelaide.edu.au (A.-L.C.); \\ wayne.boardman@adelaide.edu.au (W.B.); a1685587@student.adelaide.edu.au (R.E.) \\ * Correspondence: Farhid.hemmatzadeh@adelaide.edu.au; Tel.: +61-883-137-723
}

Received: 20 July 2020; Accepted: 22 August 2020; Published: 27 August 2020

\begin{abstract}
To determine Phascolarctid gammaherpesviruses (PhaHV) infection in South Australian koala populations, 80 oropharyngeal swabs from wild-caught and 87 oropharyngeal spleen samples and swabs from euthanased koalas were tested using two specific PCR assays developed to detect PhaHV-1 and PhaHV-2. In wild-caught koalas, active shedding of PhaHV was determined by positive oropharyngeal samples in 72.5\% (58/80) of animals, of which 44.8\% (26/58) had PhaHV-1, 20.7\% (12/58) PhaHV-2 and 34.5\% (20/58) both viral subtypes. In the euthanased koalas, systemic infection was determined by positive PCR in spleen samples and found in $72.4 \%$ (63/87) of koalas. Active shedding was determined by positive oropharyngeal results and found in $54.0 \%$ (47/87) of koalas. Koalas infected and actively shedding PhaHV-1 alone, PhaHV-2 alone or shedding both viral subtypes were $48.9 \%$ (23/47), $14.9 \%$ (7/47) and 36.2\% (17/47), respectively. Only 45.9\% (40/87) were not actively shedding, of which $40.0 \%(16 / 40)$ of these had systemic infections. Both wild-caught and euthanased koalas actively shedding PhaHV-2 were significantly more likely to be actively shedding both viral subtypes. Active shedding of PhaHV-2 had a significant negative correlation with BCS in the euthanased cohort, and active shedding of PhaHV-1 had a significant positive relationship with age in both wild-caught and euthanased cohorts.
\end{abstract}

Keywords: Phascolarctid gammaherpesvirus; koala; South Australia; PhaHV-1; PhaHV-2

\section{Introduction}

The herpesviridae family contains widely prevalent double-stranded DNA viruses, classified into three subfamilies: alphaherpesvirinae, betaherpesvirinae and gammaherpesvirinae. They have been found to infect many species across the animal kingdom, including all mammalian and avian species investigated. The persistent and often lifelong infection of herpesviruses has allowed them to co-evolve with their animal hosts, which may have led to an adaptability advantage over other infectious diseases and contributed to the survival strategy of the virus [1].

Several herpesviruses have been characterised in Australian marsupials which include Macropodid herpesvirus-1 (MaHV-1, alphaherpesvirus) detected from oral and genital mucous membrane lesions in Parma wallabies (Notamacropus parma) during a mortality event in 1975 [2] Macropodid herpesvirus-2 (MaHV-2, alphaherpesvirinae) isolated from quokka kidney cells (Setonix brachyurus) [3], Macropodid herpesvirus-3 (MaHV-3, gammaherpesvirus) and Macropodid herpesvirus-4 (MaHV-4, alphaherpesvirinae) from a variety of tissues including whole blood, mammary 
covered gammaherpesviruses, Phascolarctid gammaherpesviruses-1 [4] (PhaHV-1) and PhaHV-2, detected in the liver, spleen and nasal scrapings from various koalas (Phascolarctos cinereus) [5-7]. Recently, Stalder et al. [8] conducted a surveillance study on a range of Australian marsupials $(n=278)$ and detected six additional novel herpesviruses (one alphaherpesvirus and five gammaherpesviruses); three in common wombats (Vombatus ursinius) (VoHV1-3), one in swamp wallabies (Wallabia bicolor) (MaHV-5), one in Tasmanian devils (Sarcophilus harrisii) (DaHV-2) and one in Southern brown bandicoots (Isoodon obesulus) (PeHV-1).

Gammaherpesviruses such as Epstein Barr virus (EBV) and Kaposi sarcoma-associated herpesvirus (KHV) in humans, ovine herpesvirus-2 (OVH-2) infections in sheep and cattle, and the recently discovered novel gammaherpesviruses found in koalas (PhaHV-1 and PhaHV-2) are lymphotropic by nature, initially infecting epithelial cells and then establishing a latent infection within B-cells and T-cells which are densely populated within the host spleen and lymph nodes [9]. These viruses typically lay dormant within host lymphocytes and prevent the cell from dying via the translation of effector proteins which interfere with natural cell pathways, enabling evasion of the host's immune system. Eventually, due to external or environmental stressors, compromised immunity or other factors, a recrudescence of infection may occur causing the virus to suddenly replicate rapidly. This causes the cell to lyse, permitting new virions to be actively shed and be transmissible through respiratory and sexual transmission pathways [10-12].

Currently, PhaHV infection and prevalence has only been described in Victorian koala populations. PhaHV-1 and 2 were detected in 10.1\% (10/99) and 23.2\% (23/99) of surveyed koalas, respectively, with only one koala (1/99) being co-infected with both viral subtypes. Vaz et al. (2019) [7] also conducted a survey on 810 koalas from various populations across Victoria, with PhaHV prevalence ranging from 1 to $55 \%$. PhaHV DNA has been detected from conjunctival, nasal, oropharyngeal, cloacal and prepuce swabs [8].

The clinical significance of PhaHV is still under scrutiny, with correlations reported between PhaHV infection and "wet bottom" in koalas, a clinical manifestation of Chlamydia pecorum infection $[7,8]$. No other direct correlations between disease and PhaHV infection have been reported in koalas. Vaz et al. [5] describe severe lymphoid depletion in both lymph nodes and spleen of infected koalas. These koalas also had other comorbidities, such as chronic dermatitis caused by Sarcoptes scabiei, chronic interstitial nephritis and cystitis, bilateral conjunctivitis, pulmonary congestion, enlarged nodular spleens and airway haemorrhages [5]. Splenic lymphoid area has been reported to be positively associated with koala retrovirus (KoRV) viral loads, and disease-free koalas have been shown to have small numbers or absence of periarteriolar lymphoid sheaths or splenic lymphoid follicles [13]. Cystitis and conjunctivitis are common findings in clinical chlamydiosis [14]. Conditions observed in PhaHV-positive koalas are similar to those observed during the Macropodid herpesvirus 1 (MaHV-1) outbreak in 1975 [2], in which infected wallabies displayed signs of conjunctivitis, pneumonia, splenic and hepatic necrosis [2,3].

Given the relatively high prevalence of PhaHV in Victoria, we hypothesised that both viral subtypes occur and are actively being shed within the South Australian koala population. Moreover, we expected that a significant relationship exists between age and PhaHV infection, due to the lifelong nature of infection and the increased likelihood of infection with time. We also expected there to be a relationship between poor body condition of koalas and shedding of the virus, since stress and being immunocompromised increases the likelihood of recrudescence in other species, resulting in active shedding, including from the oropharynx [15-17]. Most gammaherpesviruses shed virions from epithelial cells, therefore DNA detected from swabs of these tissues is most likely to confirm active shedding [18]. The latent stage of infections occurs within lymphocytes, which are also densely populated within the spleen $[19,20]$, therefore infection status could be determined through DNA extracted from spleen samples.

The primary objectives of this study were to determine if either of the PhaHV viral subtypes were prevalent in South Australian koala populations, the percentage of koalas actively shedding the virus 
and whether active shedding of either viral subtype has significant correlations with factors such as age, body condition score or sex.

\section{Materials and Methods}

\subsection{Animal Ethics}

This study was approved by the University of Adelaide Animal Ethics Committee and conducted in accordance with the guideline set out in the Australian Code for the Care and Use of Animals for Scientific Purposes 8th Edition (2013) (National Health and Medical Research Council: Canberra, 2013). Animal ethics approval number for wild-caught koalas: S-2018-022 (granted 6 April 2018) and for the euthanased examination cohort: S-2016-169 (granted 9 January 2017); DEW scientific permit number: Y26054-7 (granted 7 September 2017).

\subsection{Sample Collection}

Two cohorts of koalas were used for this study: wild-caught and euthanased koalas, both from the Mount Lofty Ranges koala population in South Australia. The wild-caught cohort was considered to represent a random sample to investigate active shedding of virus, and the euthanased cohort, euthanased on welfare grounds, enabled investigations of systemic PhaHV infection status.

\subsection{Wild-Caught Koalas}

As part of a larger koala health surveillance project, wild-caught koalas were sourced from three national parks or reserves in the Mount Lofty Ranges, in Morialta, Cleland and Belair. They were caught by the flag technique, which utilized a large pole with a flag attached to the far end. The flag end of the pole was used to direct the koala down the tree. Once the koala was close to the ground, it was restrained and taken to a field hospital where it was anaesthetised and samples collected, including oropharyngeal swabs. Oropharyngeal swabs were used for PhaHV testing due to their increased rate of positivity [8] and suitability in the field. Further demographic data were recorded and included tag identification, sex, tooth wear class (TWC I-VII) [21] and body condition score (BCS 1-5) [22]. Global positioning system (GPS) data and tagged trees were recorded so koalas could be released at their point of capture. Oropharyngeal swabs were placed in sealed plastic bags and kept on ice before storage at $-80^{\circ} \mathrm{C}$ within $12 \mathrm{~h}$ of sampling. Samples were kept in the $-80^{\circ} \mathrm{C}$ freezer for up to 12 months until tested.

\subsection{Euthanased Cohort}

For koalas that had been euthanased on welfare grounds, sex, TWC [21] and BCS [22] were recorded. Oropharyngeal swabs and spleen samples were collected and stored at $-20^{\circ} \mathrm{C}$ until tested. Spleen samples were collected to determine systemic infection status due to gammaherpesvirus latency in immunological cells $[11,12,18,23,24]$.

\subsection{DNA Extraction}

DNA was extracted from both oropharyngeal swabs and spleen tissue samples using the QIAamp DNA Mini Kit (QIAGEN, Hilden, Germany). The concentration of the extracted DNA was measured using the NanoDrop One Spectrophotometer (Thermo Fisher Scientific Inc, Waltham, MA, USA). A working solution of $20 \mathrm{ng} / \mu \mathrm{L}$ of DNA from the extracted stock solutions was prepared for PCR tests.

\subsection{Quality Control}

The koala beta ( $\beta$ )-actin gene was screened via $\mathrm{qPCR}$, as a quality control, from extracted oropharyngeal and spleen DNA samples to confirm adequate DNA was extracted, adopting the same protocol described by Shojima et al. [25]. Any samples negative for $\beta$-actin were removed from the study due to a lack of quality for further testing. DNA samples were run in triplicate in a $5 \mu \mathrm{L}$ reaction. 
The DNA copy number was derived from a standard curve from the purified PCR product from a South Australian koala. Negative control contained no DNA template.

\subsection{Molecular Diagnostics (Conventional PCR)}

Specific primers were designed for PhaHV-1 and PhaHV-2 based on the published DPOL gene (Table 1), due to both viral subtypes only having a $60 \%$ nucleotide pairwise identity [6]. Primers were confirmed to be specific via NCBI Primer Blast, Sanger sequencing and by testing the primer sets on each of the PhaHV subtypes (Table 2). PCR reactions were run in $20 \mu \mathrm{L}$ of volume which included $0.5 \mu \mathrm{M}$ of forward and reverse primer, $5 \mu \mathrm{L}$ of $4 \times$ AllTaq Master Mix solution (QIAGEN, Hilden, Germany), $5 \mu \mathrm{L}$ of $20 \mathrm{ng} / \mu \mathrm{L}$ DNA template and $8.5 \mu \mathrm{L}$ of ultrapure water. PCR conditions were initial activation and denaturation of $95^{\circ} \mathrm{C}$ for $2 \mathrm{~min}$, followed by 34 cycles of denaturation at $95^{\circ} \mathrm{C}$ for $5 \mathrm{~s}$, annealing at $61{ }^{\circ} \mathrm{C}$ (PhaHV-1) or $64^{\circ} \mathrm{C}$ (PhaHV-2) for $15 \mathrm{~s}$ and extension at $72{ }^{\circ} \mathrm{C}$ for $10 \mathrm{~s}$. This was followed by a final extension step of $72{ }^{\circ} \mathrm{C}$ for $10 \mathrm{~s}$.

Table 1. PCR primers, products and annealing temperatures.

\begin{tabular}{|c|c|c|c|c|c|}
\hline Target & Primer Name & Primer Sequence & $\begin{array}{c}\text { Product } \\
\text { Bp }\end{array}$ & $\begin{array}{l}\text { Annealing } \\
\text { Temp }\end{array}$ & Reference \\
\hline \multirow{2}{*}{$\beta$-actin $(\mathrm{QC})$} & $\beta$-actin-Fwd & 5' GAGACCTTCAACACCCCAGC $3^{\prime}$ & \multirow{2}{*}{111} & \multirow{2}{*}{$60^{\circ} \mathrm{C}$} & \multirow{2}{*}{$\begin{array}{c}\text { Shojima et al. } \\
\text { (2013) [25] }\end{array}$} \\
\hline & $\beta$-actin-Rev & 5' GTGGGTCACACCATCACCAG 3' & & & \\
\hline \multirow{2}{*}{ PhaHV-1 } & VK-PhaHV-1-Fwd & 5' CGGCATCCTCCCCTGTTTAA 3' & \multirow{2}{*}{220} & \multirow{2}{*}{$61^{\circ} \mathrm{C}$} & \multirow{2}{*}{ Current study } \\
\hline & VK-PhaHV-1-Rev & 5' GCCCCTACATTCAACGAACA 3' & & & \\
\hline \multirow{2}{*}{ PhaHV-2 } & VK-PhaHV-2 Fwd & 5' CGCACTCTAAGCTGTCCCTT 3' & \multirow{2}{*}{330} & \multirow{2}{*}{$64^{\circ} \mathrm{C}$} & \multirow{2}{*}{ Current study } \\
\hline & VK-PhaHV-2 Rev & 5' TTTCGAGCATCATGCGTCCT 3' & & & \\
\hline
\end{tabular}

Table 2. Results from AGRF Sanger sequencing, showing primer sets used, sample number, type of sample (oropharyngeal or spleen), query and identity to published GenBank sequences (accession numbers: JN585829.1, JQ996387.1).

\begin{tabular}{ccccccc}
\hline \multicolumn{1}{c}{ Primers } & Sample & $\begin{array}{c}\text { PhaHV-1 } \\
\text { (JN585829.1) }\end{array}$ & $\begin{array}{c}\text { PhaHV-2 } \\
\text { (JQ996387.1) }\end{array}$ & Source & Query Cover & Per Ident \\
\hline \multirow{2}{*}{ VK-PhaHV-1 } & K18-051 & + & & Oro & $100 \%$ & $100 \%$ \\
& K18-051 & + & & Spleen & $100 \%$ & $100 \%$ \\
& K18-064 & + & & Oro & $100 \%$ & $100 \%$ \\
& K18-064 & + & + & Spleen & $100 \%$ & $100 \%$ \\
\hline VK-PhaHV-2 & K18-043 & & + & Oro & $100 \%$ & $100 \%$ \\
& K18-043 & & + & Oro & $100 \%$ & $100 \%$ \\
& K18-044 & & + & Spleen & $100 \%$ & $100 \%$ \\
\hline
\end{tabular}

\subsection{Statistical Analyses}

Binary Logistic Regression analyses (performed using IBM SPSS Statistics 23) were used to determine any significant relationships between infection of either PhaHV subtype, coinfections of PhaHV, BCS, TWC and sex. Compromised BCS was considered as BCS 1 to 3 out of $5(1-3 / 5)$ (emaciated, poor, or fair muscle condition), and good BCS as $4-5 / 5$. Variables with $p$-values of $\leq 0.05$ were considered statistically significant.

A Pearson correlation coefficient ( $\mathrm{r}$ ) analysis was conducted on both wild-caught and euthanased cohorts ( $n=80$ and $n=87$, respectively) to show correlations between covariates. Covariates analysed within each cohort were as follows; within the euthanased cohort: infected (PhaHV DNA detected in the spleen); Oro_Phas1, Oro_Phas2 and Oro_coinfection (PhaHV-1 DNA only, PhaHV-2 DNA only, or both PhaHV subtypes DNA detected from oropharyngeal swab, respectively); Spleen_Phas1, Spleen_Phas2 and Spleen_coinfection (PhaHV-1 DNA only, PhaHV-2 DNA only, or 
both PhaHV subtypes DNA detected from spleen tissue, respectively); TWC (tooth wear class) [21]; BCS (body condition score) [22], BCS_compromised (BCS 1-3/5) and sex. Covariates within the wild-caught koalas: infected (PhaHV-1 or PhaHV-2 DNA detected from oropharyngeal swab), Oro_Phas1, Oro_Phas2 and Oro_coinfection, TWC; BCS; BCS_compromised and sex. The results were visualized on a correlation heatmap using $\mathrm{R}$ version 3.0.1 using ggplot2 and ggcorrplot packages.

\section{Results}

\subsection{PhaHV-1 and PhaHV-2 Specific PCR Test}

The newly designed primer sets designated, VK-PhaHV-1 and VK-PhaHV-2, were both specific in detecting PhaHV-1 and PhaHV-2, respectively. Samples were confirmed in Sanger Sequencing (Australian Genome Research Facilities), both PhaHV-1 and PhaHV-2 showing 100\% identity to GenBank sequence identifications (Table 2) (accession numbers: JN585829.1, JQ996387.1).

\subsection{Wild-Caught Cohort of Koalas}

Approximately three-quarters, 72.5\% (58/80), of the wild-caught koalas from the Mount Lofty Ranges were actively shedding PhaHV. These were shown to be actively shedding just PhaHV-1 $(44.8 \%$ (26/58)), just PhaHV-2 (20.7\% (12/58)) or both viral subtypes (34.5\% (20/58)). Only 27.5\% (22/80) of the wild sampled koalas were not actively shedding either viral subtype (Table 3 ).

Table 3. Prevalence of active shedding of Phascolarctid gammaherpesvirus (PhaHV) viral subtypes in the two cohorts, wild-caught and euthanased.

\begin{tabular}{ccccc}
\hline \multirow{2}{*}{ Type of Infection } & \multicolumn{2}{c}{ Wild-Caught } & \multicolumn{2}{c}{ Euthanased } \\
\cline { 2 - 5 } & $\boldsymbol{n}$ & $\mathbf{\%}$ & $\boldsymbol{n}$ & $\mathbf{\%}$ \\
\hline Active Shedding & 58 & $73 \%$ & 47 & $54 \%$ \\
Active Shedding Only PhaHV-1 & 26 & $33 \%$ & 23 & $26 \%$ \\
Active Shedding Only PhaHV-2 & 12 & $15 \%$ & 7 & $8 \%$ \\
Coinfected Shedding & 20 & $25 \%$ & 17 & $20 \%$ \\
No active shedding & 22 & $28 \%$ & 40 & $46 \%$ \\
TOTAL & 80 & & 87 & \\
\hline
\end{tabular}

Infection of PhaHV-1 only had a significant positive correlation with TWC ( $p$-value $=0.023 ; \mathrm{r}=0.2$; $n=80$ ) (Figure 1A). The proportion of koalas in each TWC for each status of infection is shown in Figure 2. There were no significant associations between splenic infection with PhaHV and koalas with a compromised BCS ( $p$-value $=0.203 ; n=80)$ or with $\operatorname{sex}(p$-value $=0.776 ; n=80)$.
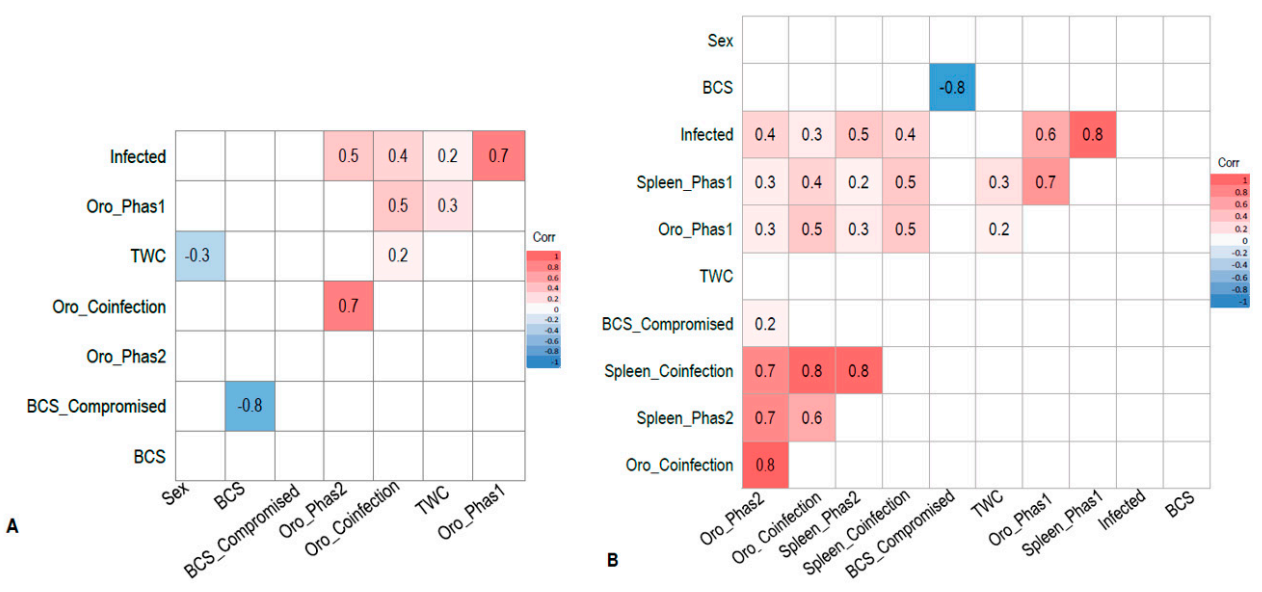

Figure 1. (A) Pearson's correlation matrix displaying interactions between covariates in wild-caught koalas from the Mount Lofty Ranges, South Australia $(n=80)$ portrayed on a heat map. Values closer to 
1.0 indicate a stronger positive correlation between the two variables. Coloured tiles contain a $p$-value $\leq 0.05$. White tiles correspond to an insignificant correlation ( $p$-value $>0.05$ ) and are excluded from the model. Variable "BCS_compromised" uses compromised koalas (BCS 1-3/5) as the reference value. (B) Pearson's correlation matrix displaying interactions between covariates from euthanased koalas sourced from various wildlife hospitals in Adelaide $(n=87)$, portrayed on a heat map. Values closer to 1.0 indicate a stronger positive correlation between the two variables. Coloured tiles contain a $p$-value $\leq 0.05$. White tiles correspond to an insignificant correlation $(p$-value $>0.05)$ and are excluded from the model. Variable "BCS_compromised" uses compromised koalas (BCS 1-3/5) as the reference value. Acronym definitions are provided in Section 2.8 (statistical analysis).

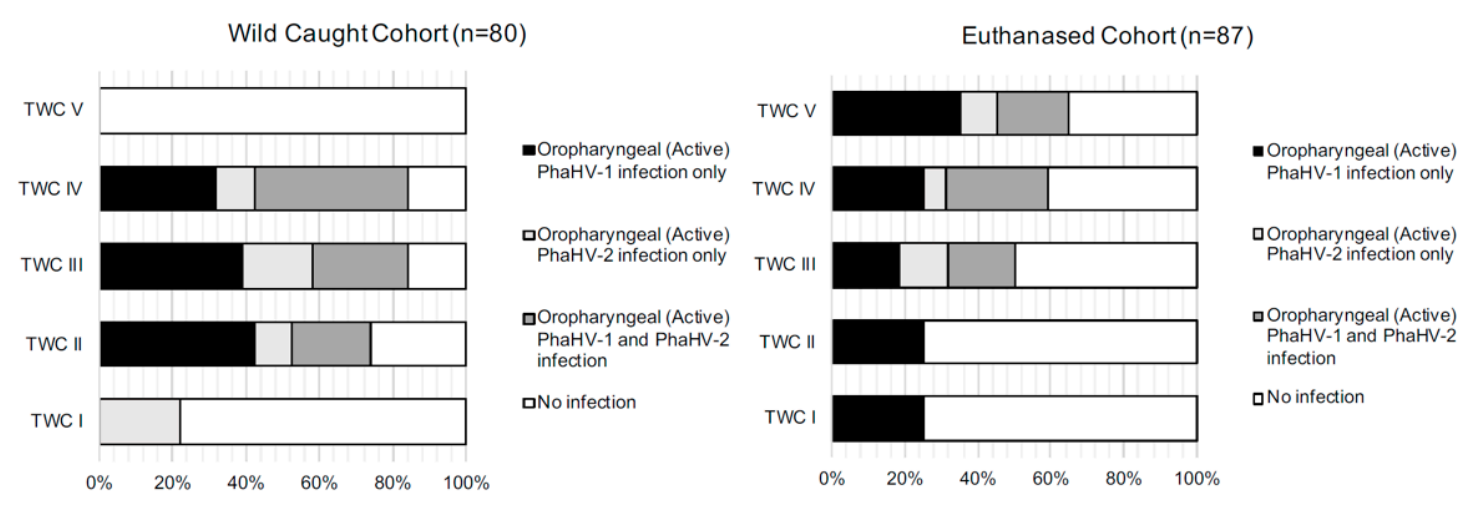

Figure 2. Histograms displaying the percentage of koalas actively shedding PhaHV-1 and PhaHV-2 and noninfected koalas within each tooth wear classification (TWC) group, for wild-caught and euthanased cohorts from South Australia.

\subsection{Euthanased Cohort of Koalas}

It was found that $72.4 \%$ (63/87) of euthanased koalas were infected with PhaHV. Active shedding of the virus occurred in $54.0 \%$ (47/87) of koalas in the euthanased cohort, with active shedding of only PhaHV-1 in 48.9\% (23/47), only PhaHV-2 in 14.9\% (7/47), or both subtypes simultaneously in $36.2 \%$ (17/47) of koalas. All koalas had matched positive spleen samples which confirmed infection (Table 3). Furthermore, $18.4 \%$ (16/87) of koalas were systemically infected with the virus but not actively shedding virus. The remaining $27.6 \%$ (24/87) of koalas were not infected at either the oropharyngeal or splenic site.

There was a strong correlation between splenic coinfection with both PhaHV-1 and PhaHV-2, as being co-infected significantly increased the likelihood of actively shedding (positive oropharyngeal sample) both viral subtypes ( $p$-value $<0.01 ; \mathrm{r}=0.80 ; n=87$ ) (Figure 1B). A significant positive correlation exists between active PhaHV-1 and PhaHV-2 infection, as koalas were 3.5 times more likely to be actively shedding PhaHV-1 if infected with PhaHV-2 ( $p$-value $=0.006 ; r=0.3 ; \operatorname{Exp}(B)=3.538)$ (Figure 1B). There was a high probability of koalas actively shedding PhaHV-1 (Exp $(B)=28.51)$ and PhaHV-2 (Exp (B) = 92.49) when co-infected with both viral subtypes in the spleen (Figure 3). Similarly, to the wild-caught koalas, the euthanased cohort had a significant positive correlation between TWC and PhaHV-1 infection (Figure 1A,B) ( $p$-value $=0.05 ; \mathrm{r}=0.2 ; n=87$ ). However, contrary to wild-caught koalas, a significant positive correlation exists between active shedding of PhaHV-2 and a compromised BCS ( $p$-value $=0.04 ; \mathrm{r}=0.2 ; n=97$ ) (Figure 1B), with euthanased koalas actively shedding PhaHV-2 being 3.5 times more likely to have a compromised BCS $(\operatorname{Exp}(B)=3.514)$. No significant association between compromised BCS and active shedding of PhaHV-1 or sex was found ( $p$-value $=0.2$ and 0.16 , respectively). 


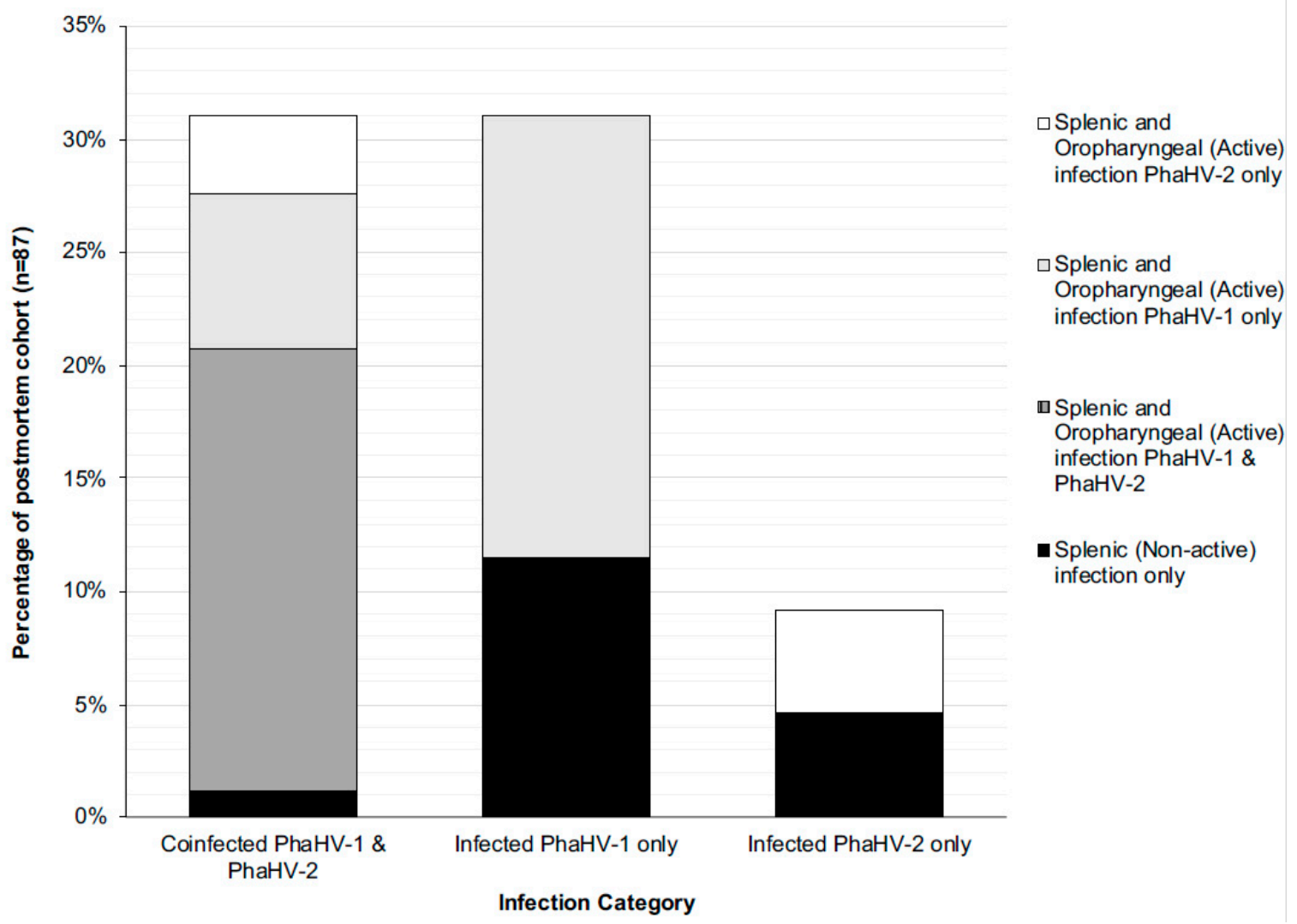

Figure 3. Histogram of infection status within the euthanased cohort $(n=87)$, displaying the following categories: coinfection with both viral subtypes (demonstrated by a dual splenic infection, and actively shedding both or singular viral subtypes, or not shedding); infected with just PhaHV-1 (demonstrated by a PhaHV-1 splenic infection and shedding or not shedding PhaHV-1); infected with just PhaHV-2 (demonstrated by a PhaHV-2 splenic infection and shedding or not shedding PhaHV-2).

\section{Discussion}

In this study, oropharyngeal and spleen samples were tested, as previous papers [5,6] succeeded in detecting and isolating the same viral subtypes from these sites. In the wild-caught cohort, only oropharyngeal swabs could be collected, whereas in the euthanased cohort, both oropharyngeal and spleen could be collected. As previously mentioned, it has been assumed that the PCR-positive oropharyngeal swabs are most likely to confirm active shedding [18], whilst latent infections occur within lymphocytes which are densely populated within the spleen $[19,20]$. In the euthanased cohort, koalas that were positive for PhaHV in both spleen tissue and oropharyngeal swab samples were deemed as infected and actively shedding the virus. Koalas only positive in the spleen were classified as being infected.

\subsection{Active Shedding}

In this study, the prevalence of PhaHV active shedding in wild-caught koalas was $72.5 \%(58 / 80)$ and was higher than that of the euthanased koalas at $54 \%$ (47/87). The rate of active shedding was higher than initially expected when compared with other herpesvirus cases found in other koalas in Australia. Stalder et al. [8] surveyed 99 captive and wild koalas in Victoria in 2015 and detected PhaHV-1 and 2 in 10.1\% (10/99) and 23.2\% (23/99) of koalas, respectively. Another study in Victoria found the prevalence of PhaHV-1 and PhaHV-2 was 17\% and 22\%, respectively, and an overall prevalence of 33\% $(n=810)$, with different populations ranging from 1 to $55 \%$ [7]. Mainland populations in Victoria had $22-46 \%$ and $31-55 \%$ test positive for PhaHV-1 and PhaHV-2 [7], respectively. These numbers are more 
consistent with the prevalence in Mount Lofty Ranges koalas, with active shedding of PhaHV-1 and PhaHV-2 at 57.5\% (46/80) and 40\% (32/80), respectively, in the wild-caught koalas and 46\% (40/87) and $27.5 \%$ (24/87), respectively, in euthanased koalas. These may represent populations with similar high densities with greater opportunity for infection spread. Differences in prevalence of viral shedding between PhaHV-1 and PhaHV-2 may show that PhaHV-2 is less established within the SA koala population than PhaHV-1, or that PhaHV-2 may have a lower tendency to actively shed than PhaHV-1. This may be due to the genetic differences between the viral subtypes, as PhaHV-2 only shares $60 \%$ pairwise identity with PhaHV-1 [6] and may contribute to higher expression of immune suppressor genes in PhaHV-2. Latency studies would need to be carried out for this theory to be concluded.

A significant relationship and positive correlation existed between active shedding of PhaHV-1 and TWC in both the wild-caught cohort $(p$-value $=0.023, \mathrm{r}=0.3)$ and the euthanased cohort $(p$-value $=0.05$, $r=0.2$ ), whereby increasing age increased the likelihood of PhaHV-1 infection. This relationship was expected: as age increased, the probability of infection increased due to the likelihood of exposure to the virus and the lifelong nature of the disease. In contrast, there was no correlation between active shedding of PhaHV-2 and TWC in either cohort. The study by Vaz et al. [7] also showed similar results regarding PhaHV-1 infection correlation with age and PhaHV-2 spread more evenly across age groups. This study proposed that PhaHV-1 infection may be more likely due to sexual contact between adult koalas, whilst PhaHV-2 infection may occur from close contact between mother and joey [7]. Vaz et al. showed repeatable detection of both PhaHV-1 and PhaHV-2 in koala cloacal regions [7,8], therefore, viral transmission between mother and joey may not only occur in the pouch, but potentially during parturition and/or from the consumption of a unique maternal faeces known as "pap". This faecal complex contains tannin protein complex-degrading enterobacteria (T-PCDE) essential for digestion of gum leaves [26] and may also be contaminated with other diseases such as Chlamydia pecorum and PhaHV-1 and PhaHV-2. Kent et al. [27] conducted a survey on sexually transmitted mustelid gammaherpesvirus 1 (MusHV-1) in European badgers (Meles meles) and investigated the prevalence of infection in adults and cubs; the high proportion of infected cubs showed a strong likelihood of vertical transmission.

Body condition score is a commonly used and effective indicator of the health status of an animal [18]. In our study, koalas with a BCS of 1-3/5 (emaciated, poor, or fair muscle condition) were considered compromised, whilst koalas with BCS 4-5/5 were considered in good condition. It was hypothesised that koalas with a low BCS were more likely to be actively shedding if infected, due to being unable to keep the herpesvirus in a latent, suppressed state. Active shedding of PhaHV-2 had a significant correlation with a compromised BCS in the euthanased koala cohort ( $p$-value $=0.04 ; \mathrm{r}=0.2 ; n=87)$, but not in the wild-caught cohort. This was likely confounded by the spread of koalas in each BCS category with a right-skewed bias towards healthy koalas in the wild-caught cohort and a more normal distribution in the euthanased cohort (Figure 4). Surprisingly, compromised BCS had no significant correlation with infection or active shedding of PhaHV-1 in either cohort. The lack of relationship between compromised BCS and active shedding of PhaHV-1 is likely due to apparently healthy animals being able to actively shed herpesviruses without developing clinical disease [10]. The potential to asymptomatically shed gammaherpesviruses or have subclinical disease has been demonstrated in other reservoir hosts [11]. In a case study of ovine herpesvirus-2 (OvHV-2; gammaherpesvirus), Li et al. [10] took a series of nasal swabs and discovered that healthy lambs between 6 and 9 months of age had an active shedding prevalence of $61 \%(n=56)$. Gammaherpesviruses can be more likely to cause disease in susceptible, nonreservoir hosts, as seen with cattle infected with OvHV-2 causing malignant catarrhal fever (MCF) [28]. 


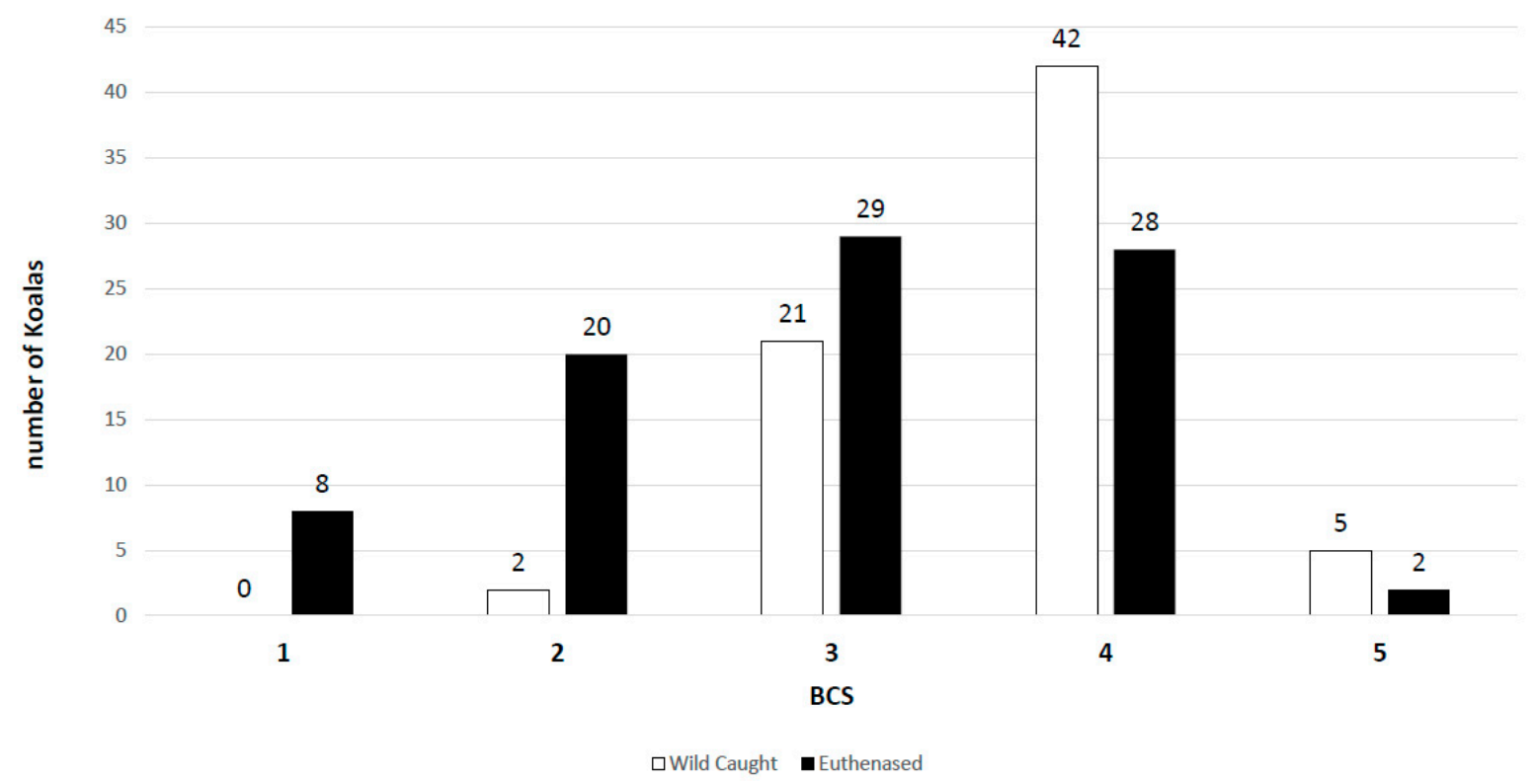

Figure 4. Histogram displaying the distribution of koalas in each cohort within their assigned body condition score (BCS).

\subsection{Systemic Infection and Active Shedding}

The majority of infected euthanased koalas were actively shedding the virus $(74.6 \% ; 47 / 63)$, whilst only $25.4 \%(16 / 63)$ of infected koalas were not actively shedding. There was a strong positive correlation between splenic coinfection of both viral subtypes and active shedding of the virus $(0.8)$ (Figure 1B). In the euthanased cohort, koalas infected with PhaHV-2 were 3.5 times more likely to be coinfected with PhaHV-1 $(\operatorname{Exp}(B)=3.538 ; p$-value $=0.006 ; n=87)$ and as a result were 1.5 times more likely to be actively shedding PhaHV-1 with every incremental increase in TWC $(\operatorname{Exp}(\mathrm{B}))=1.514$; $p$-value $=0.05 ; n=87$ ). This suggests that viral coinfection highly predisposes koalas to active shedding of the virus. Potentially, increased expression of viral effector proteins from both PhaHV-1 and PhaHV-2 could lead to greater immunosuppression, initiating recrudescence. A study investigating herpesvirus coinfection in humans showed that infection of EBV and HHV-7 were also shown to promote HHV-6 infection and disease [29-32] however, this may just be a result of severe immunosuppression that triggers viral coinfection, as stated by Handous et al. [33]. PhaHV-2 had a significant correlation with a compromised BCS, suggesting that PhaHV-2 may be an indicator of other underlying health conditions such as immunosuppression, stress and coinfection of other diseases including PhaHV-1, Chlamydia pecorum or koala retrovirus.

In our study, there was no significant relationship between sex and infection status. Yet Stalder et al. [8] showed a significant relationship between infection and sex, with male koalas more likely to have an infection. Vaz et al. [7] showed that females without young were more likely to be infected than those with young; they also found a correlation between PhaHV and "wet bottom", a clinical sign of overt chlamydiosis. The association of potentially infertile females with PhaHV may be confounded by chlamydial infection, a known cause of reproductive pathology in koalas [34].

\subsection{Sites of Active Viral Shedding}

In our study, most oropharyngeal swabs from infected (spleen positive) koalas were positive for Phascolarctid gammaherpesvirus, suggesting that significant active shedding is from rostral epithelial tissues (nasal, oropharyngeal). This finding differs to that of Vaz et al. [7], which found that rostral (ocular, nasal, oropharyngeal) swabbing was less likely to pick up an infection in comparison with caudal (urogenital, cloacal) swabbing. Samples from caudal epithelial tissues were not tested in our 
study, therefore, shedding from different epithelial sites needs to be investigated further to provide tissue tropism insights. One concern about the interpretation of active shedding is the contamination of the oropharyngeal swabs with cells within which latency has been established, that is, circulating mononucleocytes. Nevertheless, due to the tissue tropism of herpesviruses, it is less likely to detect virus in epithelial swabs when the animals are only infected in latent forms $[15,35,36]$. It is also highly likely that the detected viruses in epithelial cells are actively shed viruses [37].

\subsection{Potential Areas for Further Research}

This study focused on the presence and prevalence of Phascolarctid herpesviruses within the mainland South Australian koala population. Further research is needed to understand the association of PhaHV with other infections-primarily koala retrovirus (KoRV) and Chlamydial infection-since PhaHV may be playing a role in the augmentation of clinical disease or be shed secondary to them. Since PhasHV-2 had an association to koalas with a compromised BCS and increased PhasHV-1 shedding, this could be suggestive of an increase in pathogenicity in this subtype. Investigation into disease presentations, haematology and immune function markers with and without PhaHV infection may shed some light on the effect of these viruses. Serological studies could be conducted to investigate the koalas' response to PhaHV infection, since the koalas' immune system has often been perceived as "immunologically lazy" [38,39]. These studies would help to further determine the clinical significance, host response to infection and impact of these newly discovered viruses on koala populations.

\section{Conclusions}

We showed there was a high prevalence of PhaHV infection in koalas in the Mount Lofty Ranges population, with more than two-thirds of both wild-caught and euthanased cohorts actively shedding the virus. PhaHV-1 had a greater prevalence within the SA koala populations, with more koalas actively shedding PhaHV-1 than PhaHV-2.

Despite being less prevalent, koalas coinfected with PhaHV-2 were more likely to also be infected and actively shedding PhaHV-1. Neither of the viral subtypes were shown to have any significant relationship with BCS in wild-caught koalas; however, PhaHV-2 infection had a significant correlation with BCS in euthanased koalas. PhaHV-1 infection was also shown to be positively correlated with TWC in both cohorts, whilst sex had no significant correlation with either viral subtype in both cohorts.

The clinical significance of these recently discovered Phascolarctid herpesviruses is still unknown, and additional investigation into the pathogenicity, clinical signs of the virus and coinfection with other pathogens is important. Uncovering the significance of PhaHV will help determine the health status and guide the management of koala populations.

Author Contributions: Conceptualization, F.H. and T.S.; methodology, V.K., T.S., N.S.; W.B., R.E.; software, A.-L.C., V.K.; formal analysis, A.-L.C., V.K.; investigation, F.H., A.-L.C., V.K., N.S.; W.B., and T.S.; resources, F.H., N.S.; data curation, A.-L.C.; writing—original draft preparation, V.K.; writing-review and editing, F.H., A.-L.C., V.K., T.S., N.S.; W.B.; visualization, F.H., A.-L.C., V.K.; supervision, F.H.; project administration, F.H., N.S.; funding acquisition, F.H., N.S. All authors have read and agreed to the published version of the manuscript.

Funding: This research was funded by The University of Adelaide HDR support fund grant number 13107182.

Acknowledgments: We thank Kandarp Patel (The University of Adelaide) for advising us on statistical analysis and modelling, Adelaide Zoo and the Department of Environment and Water, SA for koala capture fieldwork, Adelaide Koala and Wildlife Hospital, Adelaide and Adelaide Hills based veterinary clinics, SA Fauna Rescue, Adelaide Koala Rescue, Adelaide and Adelaide Hills Koala Rescue and other fauna rescue groups for their help in provision of koalas.

Conflicts of Interest: The authors declare no conflict of interest. The funders had no role in the design of the study; in the collection, analyses, or interpretation of data; in the writing of the manuscript, or in the decision to publish the results. 


\section{References}

1. Chapter 9 - Herpesvirales. In Fenner's Veterinary Virology (Fifth Edition); MacLachlan, N.J., Dubovi, E.J., Eds.; Academic Press: Boston, MA, USA, 2017. [CrossRef]

2. Finnie, E.P.; Littlejohns, I.R.; Acland, H.M. Letter: Mortalities in parma wallabies (Macropus parma) associated with probable herpesvirus. Aust. Vet. J. 1976, 52, 294. [CrossRef] [PubMed]

3. Johnson, M.A.; Whalley, J.M.; Littlejohns, I.R.; Dickson, J.; Smith, V.W.; Wilks, C.R.; Reisner, A.H. Macropodid herpesviruses 1 and 2: Two herpesviruses from Australian marsupials differentiated by restriction endonucleases, DNA composition and hybridization. Brief Rep. Arch. Virol. 1985, 85, 313-319. [CrossRef] [PubMed]

4. Smith, J.A.; Wellehan, J.F., Jr.; Pogranichniy, R.M.; Childress, A.L.; Landolfi, J.A.; Terio, K.A. Identification and isolation of a novel herpesvirus in a captive mob of eastern grey kangaroos (Macropus giganteus). Vet. Microbiol. 2008, 129, 236-245. [CrossRef] [PubMed]

5. Vaz, P.; Whiteley, P.L.; Wilks, C.R.; Duignan, P.J.; Ficorilli, N.; Gilkerson, J.R.; Browning, G.F.; Devlin, J.M. Detection of a novel gammaherpesvirus in koalas (Phascolarctos cinereus). J. Wildl. Dis. 2011, 47, 787-791. [CrossRef] [PubMed]

6. Vaz, P.; Whiteley, P.L.; Wilks, C.R.; Browning, G.F.; Gilkerson, J.R.; Ficorilli, N.; Devlin, J.M. Detection of a second novel gammaherpesvirus in a free-ranging koala (Phascolarctos cinereus). Wildl. Dis. 2012, 48, 226-229. [CrossRef]

7. Vaz, P.K.; Legione, A.R.; Hartley, C.A.; Devlin, J.M. Detection and Differentiation of Two Koala Gammaherpesviruses by Use of High-Resolution Melt (HRM) Analysis Reveals Differences in Viral Prevalence and Clinical Associations in a Large Study of Free-Ranging Koalas. J. Clin. Microbiol. 2019, 57. [CrossRef]

8. Stalder, K.; Vaz, P.K.; Gilkerson, J.R.; Baker, R.; Whiteley, P.; Ficorilli, N.; Tatarczuch, L.; Portas, T.; Skogvold, K.; Anderson, G.A.; et al. Prevalence and Clinical Significance of Herpesvirus Infection in Populations of Australian Marsupials. PLoS ONE 2015, 10, e0133807. [CrossRef]

9. Sugden, B.; Kintner, C.R.; Mark, W. The molecular biology of lymphotropic herpesviruses. Adv. Cancer Res. 1979, 30, 239-278. [CrossRef]

10. Li, H.; Taus, N.S.; Lewis, G.S.; Kim, O.; Traul, D.L.; Crawford, T.B. Shedding of ovine herpesvirus 2 in sheep nasal secretions: The predominant mode for transmission. J. Clin. Microbiol. 2004, 42, 5558-5564. [CrossRef]

11. Ackermann, M. Pathogenesis of gammaherpesvirus infections. Vet. Microbiol. 2006, 113, 211-222. [CrossRef]

12. Hussy, D.; Janett, F.; Albini, S.; Stauber, N.; Thun, R.; Ackermann, M. Analysis of the pathogenetic basis for shedding and transmission of ovine gamma herpesvirus 2. Clin. Microbiol. 2002, 40, 4700-4704. [CrossRef] [PubMed]

13. Fabijan, J.; Sarker, N.; Speight, N.; Owen, H.; Meers, J.; Simmons, G.; Seddon, J.; Emes, R.D.; Tarlinton, R.; Hemmatzadeh, F.; et al. Pathological Findings in Koala Retrovirus-positive Koalas (Phascolarctos cinereus) from Northern and Southern Australia. J. Comp. Pathol. 2020, 176, 50-66. [CrossRef]

14. Hemsley, S.; Canfield, P.J. Histopathological and immunohistochemical investigation of naturally occurring chlamydial conjunctivitis and urogenital inflammation in koalas (Phascolarctos cinereus). J. Comp. Pathol. 1997, 116, 273-290. [CrossRef]

15. Flano, E.; Kim, I.J.; Woodland, D.L.; Blackman, M.A. Gamma-herpesvirus latency is preferentially maintained in splenic germinal center and memory B cells. J. Exp. Med. 2002, 196, 1363-1372. [CrossRef] [PubMed]

16. Gerow, C.M.; Rapin, N.; Voordouw, M.J.; Elliot, M.; Misra, V.; Subudhi, S. Arousal from hibernation and reactivation of Eptesicus fuscus gammaherpesvirus (EfHV) in big brown bats. Transbound. Emerg. Dis. 2019, 66, 1054-1062. [CrossRef] [PubMed]

17. Weck, K.E.; Barkon, M.L.; Yoo, L.I.; Speck, S.H. Mature B cells are required for acute splenic infection, but not for establishment of latency, by murine gammaherpesvirus. Virology 1996. [CrossRef]

18. Feldman, E.R.; Kara, M.; Coleman, C.B.; Grau, K.R.; Oko, L.M.; Krueger, B.J.; Renne, R.; van Dyk, L.F.; Tibbetts, S.A. Virus-encoded microRNAs facilitate gammaherpesvirus latency and pathogenesis in vivo. mBio 2014, 5, e00914-e00981. [CrossRef]

19. Sharma, V.; Mobeen, F.; Prakash, T. Comparative Genomics of Herpesviridae Family to Look for Potential Signatures of Human Infecting Strains. Int. J. Genom. 2016, 2016, 9543274. [CrossRef]

20. van Dyk, L.F.; Virgin, H.W.t.; Speck, S.H. Maintenance of gammaherpesvirus latency requires viral cyclin in the absence of B lymphocytes. Virology 2003, 77, 5118-5126. [CrossRef] 
21. Martin, R.W. Age-Specific Fertility in Three Populations of the Koala, Phascolarctos cinereus Goldfuss, in Victoria. Wildl. Res. 1981, 8, 275-283. [CrossRef]

22. Blanshard, W.H.; Bodley, K. Chapter 8: Koalas. In Medicine of Australian Mammals, 1st ed.; Vogelnest, L., Woods, R., Eds.; CSIRO Publishing: Collingwood, Clayton, Australia, 2008; pp. 227-328.

23. Lieberman, P.M. Keeping it quiet: Chromatin control of gammaherpesvirus latency. Nat. Rev. Microbiol. 2013, 11, 863-875. [CrossRef] [PubMed]

24. King, A.; Adams, M.J.; Carstens, E.B.; Lefkowitz, E.J. Virus Taxonomy: Ninth Report of the International Committee on Taxonomy of Viruses. Elsevier 2012,99-107. [CrossRef]

25. Shojima, T.; Yoshikawa, R.; Hoshino, S.; Shimode, S.; Nakagawa, S.; Ohata, T.; Nakaoka, R.; Miyazawa, T. Identification of a novel subgroup of Koala retrovirus from Koalas in Japanese zoos. Virology 2013, 87, 9943-9948. [CrossRef] [PubMed]

26. Osawa, R.; Blanshard, W.H.; Ocallaghan, P.G. Microbiological Studies of the Intestinal Microflora of the Koala, Phascolarctos cinereus. Pap, a Special Maternal Feces Consumed by Juvenile Koalas. Aust. J. Zool. 1993, 41, 527-536. [CrossRef]

27. Kent, A.; Ehlers, B.; Mendum, T.; Newman, C.; Macdonald, D.W.; Chambers, M.; Buesching, C.D. Genital tract screening finds widespread infection with mustelid gammaherpesvirus 1 in the uropean badger (meles meles). J. Wildl. Dis. 2018, 133-137. [CrossRef]

28. Meier-Trummer, C.S.; Rehrauer, H.; Franchini, M.; Patrignani, A.; Wagner, U.; Ackermann, M. Malignant catarrhal fever of cattle is associated with low abundance of IL-2 transcript and a predominantly latent profile of ovine herpesvirus 2 gene expression. PLoS ONE 2009, 4. [CrossRef]

29. Razonable, R.R.; Brown, R.A.; Humar, A.; Covington, E.; Alecock, E.; Paya, C.V. Herpesvirus infections in solid organ transplant patients at high risk of primary cytomegalovirus disease. J. Infect. Dis. 2005, 192, 1331-1339. [CrossRef]

30. Blazquez-Navarro, A.; Dang-Heine, C.; Wittenbrink, N.; Bauer, C.; Wolk, K.; Sabat, R.; Westhoff, T.; Sawitzki, B.; Reinke, P.; Thomusch, O.; et al. BKV, CMV, and EBV Interactions and their Effect on Graft Function One Year Post-Renal Transplantation: Results from a Large Multi-Centre Study. EBioMedicine 2018, 34, 113-121. [CrossRef]

31. Aalto, S.M.; Linnavuori, K.; Peltola, H.; Vuori, E.; Weissbrich, B.; Schubert, J.; Hedman, K. Immunoreactivation of Epstein-Barr virus due to cytomegalovirus primary infection. J. Med. Virol. 1998, 56, 186-191. [CrossRef]

32. Jain, R.; Trehan, A.; Mishra, B.; Singh, R.; Saud, B.; Bansal, D. Cytomegalovirus disease in children with acute lymphoblastic leukemia. J. Pediatric Hematol./Oncol. 2016, 33, 239-247. [CrossRef]

33. Handous, I.; Achour, B.; Marzouk, M.; Rouis, S.; Hazgui, O.; Brini, I.; Khelif, A.; Hannachi, N.; Boukadida, J. Co-infections of human herpesviruses (CMV, HHV-6, HHV-7 and EBV) in non-transplant acute leukemia patients undergoing chemotherapy. Virology 2020, 17, 37. [CrossRef] [PubMed]

34. Robbins, A.; Hanger, J.; Jelocnik, M.; Quigley, B.L.; Timms, P. Longitudinal study of wild koalas (Phascolarctos cinereus) reveals chlamydial disease progression in two thirds of infected animals. Sci. Rep. 2019, 9, 13194. [CrossRef] [PubMed]

35. Collins, C.M.; Boss, J.M.; Speck, S.H. Identification of infected B-cell populations by using a recombinant murine gammaherpesvirus 68 expressing a fluorescent protein. Virology 2009, 83, 6484-6493. [CrossRef] [PubMed]

36. Rekow, M.M.; Darrah, E.J.; Mboko, W.P.; Lange, P.T.; Tarakanova, V.L. Gammaherpesvirus targets peritoneal B-1 B cells for long-term latency. Virology 2016, 492, 140-144. [CrossRef] [PubMed]

37. Usherwood, E.J.; Roy, D.J.; Ward, K.; Surman, S.L.; Dutia, B.M.; Blackman, M.A.; Stewart, J.P.; Woodland, D.L. Control of gammaherpesvirus latency by latent antigen-specific CD8(+) T cells. J. Exp. Med. 2000, 192, 943-952. [CrossRef]

38. Higgins, D.; Lau, Q.; Maher, I. Koala immunology and the koala retrovirus (KoRV). Tech. Rep. Aust. Mus. Online 2014, 35-38. [CrossRef]

39. Wilkinson, R.; Kotlarski, I.; Barton, M. Koala lymphoid cells: Analysis of antigen-specific responses. Vet. Immunol. Immunopathol. 1992, 33, 237-247. [CrossRef]

(C) 2020 by the authors. Licensee MDPI, Basel, Switzerland. This article is an open access article distributed under the terms and conditions of the Creative Commons Attribution (CC BY) license (http://creativecommons.org/licenses/by/4.0/). 